\title{
Progress in the Use of Molecular Hydrogen for Cancer Treatment
}

\author{
Dong Yue Du, Yun Hua Peng, Jian Kang Liu, Jian Gang Long \\ Center for Mitochondrial Biology and Medicine, Biomedical Information Engineering Laboratory of State Ministry of Education, \\ School of Life Science and Technology, Xi'an Jiaotong University, Xi'an 710049, China
}

\begin{abstract}
Molecular hydrogen is an effective antioxidant. Numerous studies have demonstrated the therapeutic effects of hydrogen in the treatment of various human diseases. The possibility of using hydrogen in the treatment of cancer was first discovered in 1975, and in recent studies, researchers have reported numerous positive effects of hydrogen in cancer therapy, including: 1) the alleviation of complications caused by chemotherapy; 2) a reduction of complications caused by radiotherapy; 3) delays in the progression of cancer; and 4) enhanced efficacy of conventional therapy when used in combination with hydrogen. This article reviews the research progress in the use of hydrogen in the treatment of cancer, and proposes future directions for research in this field.
\end{abstract} of life

Key words: Molecular hydrogen; Cancer; Chemotherapy; Radiotherapy; Anticancer drug; Complications; Quality

\section{Introduction}

Hydrogen is the smallest molecule, and is a colorless, tasteless, odorless, and nontoxic gas at ambient temperatures [1]. Over the past few decades, there has been extensive researches on the beneficial effects of hydrogen in the clinical setting, including studies of its anti-oxidative, anti-inflammatory and potential anti-cancer activity [2].

The possibility of using hydrogen in the treatment of cancer was first described by Dore M et al. in 1975. They reported that hyperbaric hydrogen treatment for 2 weeks could lead to significant regression of skin carcinomas in mice [3]. However, concerns about the safety of transporting, storing and administering hyperbaric hydrogen limited the application of this therapy. However, in 2007, Ohsawa I et al. suggested that inhaling small amounts of hydrogen gas could selectively reduce the level of oxygen radicals, and could protect neurons from injury caused by ischemia-reperfusion or inflammation [4]. Since then, the potential therapeutic effects of molecular hydrogen have attracted increasing attention. Several studies have shown that hydrogen has positive effects against metabolic syndrome, inflammation, injury, and cancer [5].

The application of hydrogen for cancer treatment is now a hot research topic. Clinical and experimental research has indicated that hydrogen could reduce complications associated with treatment using conventional anti-cancer drugs, could prevent cancer progression and could improve the quality of life of cancer patients. In this article, we review the progress made in the application of hydrogen for the prevention and treatment of cancer, and propose future

Corresponding author: Jian Gang Long, PhD, Center for Mitochondrial Biology and Medicine, School of Life Science and Technology, Xi'an Jiaotong University, 28 West Xianning Road, Xi'an 710049, China; Tel: +86 1992907 2355; Email: jglong@mail.xjtu.edu.cn directions for research on the potential use of molecular hydrogen for cancer therapy.

\section{Hydrogen Reduces the Complications Associ- ated with Chemotherapy}

Chemotherapy is commonly used for most types of cancer, as has been the mainstay of treatment for decades. However, these antitumor drugs are often cytotoxic, and also cause damage to normal cells and organs [6], potentially leading to complications such as nephrotoxicity, cardiotoxicity and hepatotoxicity. These side effects strongly decrease the life quality of cancer patients.

Studies have found that human tumor cells produce more reactive oxygen species (ROS) than normal cells, thus promoting the proliferation, angiogenesis, and DNA synthesis of cancer cells [7]. Nakashima-Kamimura $\mathrm{N}$ et al. found that inhaling hydrogen or drinking hydrogen-rich water, which is water contains a high concentration of dissolved hydrogen, could alleviate the nephrotoxicity caused by cisplatin, an anti-cancer drug, without affecting the drug's anti-tumor activity [8].

Doxorubicin (DOX) is widely used as an anticancer drug, but its cardiotoxicity and hepatotoxicity limit its application [9]. It was reported that hydrogen-rich saline treatment could effectively inhibit the heart and liver injury, as well as the inflammation caused by DOX [9]. Gefitinib is an epidermal growth factor receptor (EGFR) tyrosine kinase inhibitor, which is an effective drug used in the treatment of non-small cell lung cancer. However, it can cause acute lung injury [10]. Terasaki $\mathrm{Y}$ et al. recently reported that hydrogen water intake could effectively protect the lungs of mice from severe damage caused by gefitinib without interfering with the anti-tumor activity of the drug [10]. Hydrogen water also alleviated the liver injury caused by mFOLFOX6 chemotherapy in colorectal cancer patients [6]. 
The above studies suggest that hydrogen can improve the life quality of cancer patients during chemotherapy by mitigating the side effects of anti-cancer drugs. However, most of these studies were based on animal and cell models, and little has been reported regarding the possible molecular mechanisms. Therefore, more clinical evidence and molecular experiments are needed to verify the impact of hydrogen on chemotherapy-associated complications.

\section{Hydrogen Reduces Radiotherapy-related Complications}

Radiation therapy is also frequently used in treating cancer, but the radiation-induced complications limit the doses that can be administrated and reduce the quality of life of patients. During treatment with ionizing radiation treatment, oxidative injuries which produce ROS are the major mediators of radiation-induced complications [11]. It has been reported that hydrogen could ameliorate radiation-induced complications during cancer treatment by selectively reducing the level of free radicals [12]. In 2011, Chuai Y et al. first proposed that a hydrogen-rich solution could be used during radiotherapy to prevent the development of radiation pneumonitis [12].

Radiation therapy also causes gastrointestinal toxicity. Xiao HW et al. found that hydrogen water ameliorated the radiation-induced intestinal injury, and increased the survival rate and body weight of experimental mice [13]. Clinical research has shown similar results. For example, Kang KM et al. found that drinking hydrogen-rich water could improve the life quality of liver tumor patients receiving radiotherapy by reducing the biological reaction to radiation-induced oxidative stress without compromising the anti-tumor effects of treatment [14].

In 2014, Mei K et al. [15] found that injecting hydrogen water could significantly reduce the development of radiation-induced dermatitis after single and divided irradiation of the neck and head in rats. They also found that hydrogen-rich medium could protect human immortalized epidermal $(\mathrm{HaCaT})$ cells from radiation-induced injury by significantly reducing the oxygen level and increasing the antioxidative capacity of the cells. In 2018, it was reported that hydrogen may protect $\mathrm{HaCaT}$ cells from UVB radiation-induced oxidative stress by inhibiting the activation of Nrf2/HO-1 through the PI3K/Akt pathway [16].

Another hydrogen-producing nanomaterial, $\mathrm{PdH} 0.2$ nanocrystals, have been reported to release hydrogen and heat in a controlled manner, with tumor-targeted delivery. These nanocrystals could produce hydrogen locally and have been found to reduce side effects and enhance the positive effects of hyperthermia in different cancer cell lines [17].

It was also found that hydrogen-rich water was effective in preventing osteoradionecrosis of the jaw, which is a serious complication of radiotherapy during the treatment of head and neck cancer patients [18].

The above studies indicate that hydrogen has significant effects that can mitigate different complications caused by radiotherapy. Different methods of hydrogen intake have been demonstrated to be effective, and clinical results have shown that hydrogen could improve the quality of in cancer. Although researchers have demonstrated the effectiveness of hydrogen and its ability to reduce the ROS level, only one study so far has discussed the possible mechanism by which hydrogen protects cells from radiation-induced oxidative stress [16]. Therefore, the molecular mechanisms should be a focus of further investigation.

\section{Hydrogen Delays the Progression of Cancer}

Besides reducing complications during cancer treatment, hydrogen was also found to have beneficial effects against cancer progression. In 2008, Saitoh Y et al. first proposed that hydrogen water may inhibit tumor growth based on observations in human tongue carcinoma cells and fibrosarcoma cells [19].

Oxidative stress is considered to be a strong contributor to the progression from fatty liver to nonalcoholic steatohepatitis (NASH), and even hepatocarcinogenesis. It was reported that hydrogen water was effective in the treatment of NASH in mice, and may prevent the progression of hepatocarcinogenesis [20].

In 2017, Li Q et al. reported hydrogen-occluding-silica (H2-silica), a novel hydrogen-generating material which could release hydrogen into the medium. The H2-silica could effectively inhibit the proliferation and migration of human esophageal squamous cell carcinoma (KYSE-70) cells. They suggested that the $\mathrm{H} 2$-silica may selectively increase cell apoptosis by triggering the non-caspase pathway, because the $\mathrm{Bax} / \mathrm{Bcl}-2$ ratios were increased specifically in cancer cell lines [21].

Loss of immunological activity of differentiation cluster $\mathrm{CD}^{+} \mathrm{T}$ cells is associated with the inactivation of peroxisome proliferator-activated receptor gamma coactivator 1 alpha (PGC-1 $\alpha$ ), which causes mitochondrial dysfunction, and is widely found in patients with advanced cancer [22]. In 2018, Akagi J et al. found that hydrogen gas could activate PGC-1 $\alpha$ and restore the exhausted $\mathrm{CD} 8^{+} \mathrm{T}$ cells in stage IV colorectal carcinoma patients, suggesting that hydrogen could improve the prognosis of patients with advanced cancer [22].

Recently, Wang D et al. found that hydrogen gas inhibited the viability, migration and invasion of lung cancer cells in vitro; and inhibited tumor growth in vivo [23]. They also discovered that hydrogen inhibits lung cancer progression by down-regulating the expression and structural stability of structural maintenance of chromosomes 3 (SMC3). Their study showed that hydrogen could down-regulate the expression of SMC3, structural maintenance of chromosomes 5 (SMC5), structural 
maintenance of chromosomes 6 (SMC6) and Nipped-B homolog (Drosophila) (NIBPL). They found that hydrogen translocated SMC3 during cell division, resulting in decreased cell stability and enhanced SMC3 ubiquitination in A549 and H1975 lung cancer cell lines [23]. This study suggested that hydrogen gas may inhibit the progression of lung cancer by down-regulating SMC3.

These research studies showed that hydrogen may delay cancer progression by inhibiting the growth of tumor cells and triggering tumor cell apoptosis. Some possible pathways have been identified, including the non-caspase apoptosis pathway, and key proteins such as PGC- $1 \alpha$ and SMC3. Some of these studies suggested hydrogen may interfere with different proteins located in the nucleus and cytoplasm, indicating that hydrogen may participate in the activation or inhibition of multiple pathways. However, more experiments are needed to support the role of hydrogen in delaying cancer progression, and to determine whether this finding translates to the clinic.

\section{Combination Treatment with Hydrogen and Anti-Cancer Drugs}

It was also reported that using hydrogen in combination with other anti-cancer drugs appeared to be useful in enhancing the therapeutic effects of the drugs.
For example, Runtuwene $\mathrm{J}$ et al. found that hydrogen water could significantly increase the survival rate, improve the anti-oxidative effects and enhance the pro-apoptotic effects of 5-FU in a mouse model of colon cancer [2].

The PI3K/AKT signaling pathway has proven to be a viable target for novel antitumor drugs [16]. Researchers recently found that the combination of hydrogen-rich saline with a PI3K inhibitor, LY294002, could reduce proliferation and promote apoptosis in A549 non-small cell lung cancer cells [24]. Although these findings are preliminary, they suggest that combining hydrogen with other treatments may have additive or synergistic effects, in addition to decreasing treatment-related toxicity.

\section{Conclusion}

Molecular hydrogen has anti-oxidative, anti-inflammatory and anti-allergic effects mediated by its selective removal of free radicals. Since 2007, when safer and more convenient methods of hydrogen intake were proven to be effective in the treatment of disease [4], numerous studies have examined the potential of using hydrogen for a variety of diseases and conditions.

The biological effects of hydrogen in disease models and the potential mechanisms of action of hydrogen in these diseases have also been investigated in our lab. In 2016, we

Colon Cancer
- Decrease cancel progression with 5-FU
- Inhibit liver injury caused by chemotherapy
- Restore exhausted CD8 ${ }^{+}$T cells
Non-small Cell Lung Cancer
- Decrease cancer progression
- Improve effects of anti-cancer drugs
- Ameliorate lung damage caused by
chemotherapy
- Decrease tumor growth
Liver Cancer
- Decrease cancer progression from fatty
liver disease
- Ameliorate radiation-induced oxidative
stress $\quad$ Tead and Neck Cancer
- Ameliorate osteoradionecrosis of the jaw
caused by radiotherapy
- Reduce radiation-inducecl dermatitis

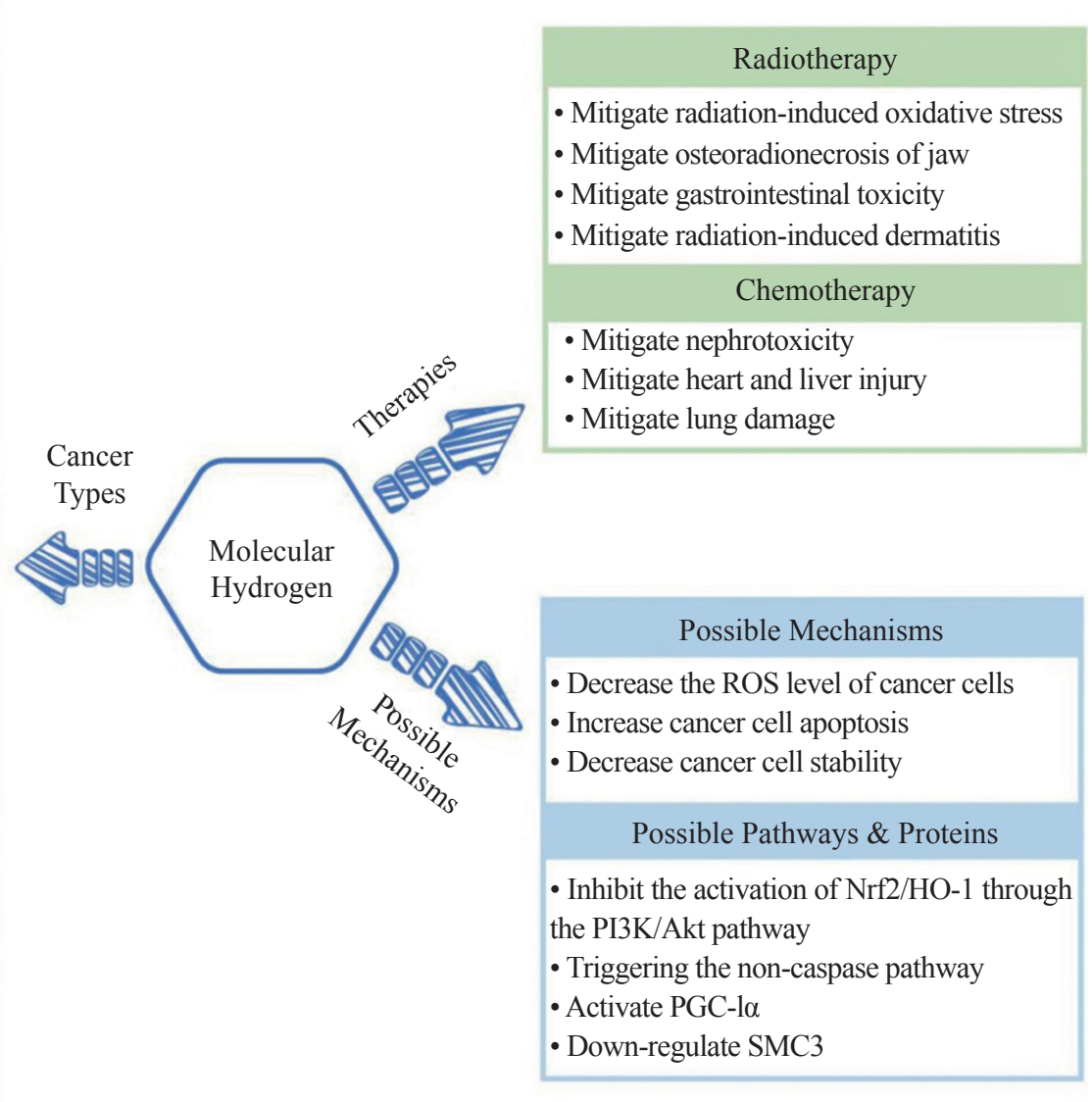

Figure 1 Current progress on the use of hydrogen in the treatment of cancer. 
found that inhalation of a small amount of hydrogen could improve the cardiac function and neurological outcomes in the asphyxia rat model of cardiac arrest, and the therapeutic benefits were superior to traditional hypothermia methods [25]. It was also noted that coral calcium hydride (CCH), an efficient hydrogen-releasing agent, could effectively prevent high fat diet-induced nonalcoholic fatty liver disease by improving the mitochondrial function and activating phase II enzymes [26]. Recently, we discovered that hydrogen may gender-dependently improve the cognitive function of APP/ PSI mice (a model of Alzheimer's disease) by interfering with the estrogen receptor- $\beta$ and brain-derived neurotrophic factor (ER $\beta$-BDNF) signaling pathways [27]. The medical effects of hydrogen are gaining increasing interest, and numerous investigators are evaluating the effects of hydrogen in various disease states and are working to determine the associated mechanisms of action.

Because cancer cells produce more ROS than normal cells, and since hydrogen can act as an anti-oxidative molecule, hydrogen may be useful for cancer treatment. In fact, several studies have already indicated that hydrogen has anti-tumor effects that are at least partially mediated through reductions of the ROS in tumor cells to suppress cell growth $[28,29]$.

Nevertheless, there is only limited information available about the optimal doses and timing of hydrogen therapy, as well as the safety of this treatment [30]. Moreover, there have been only a few studies of the possible molecular mechanisms underlying the effects of hydrogen. Therefore, further studies are needed to elucidate the mechanism(s) of action when hydrogen is used alone and in combination with other agents; and more clinical research is also needed to confirm the safety and efficacy of the treatment.

In conclusion, recent studies have found that hydrogen treatment could alleviate the adverse effects induced by chemotherapy and radiotherapy to improve the quality of life of cancer patients. Hydrogen treatment may also slow down the progression of cancer; and the combined use of hydrogen with other anti-cancer drugs may enhance the anti-cancer effects of the treatment. Some possible molecular mechanisms by which hydrogen induces these effects have been described. In Figure 1, we summarize the current progress that has been made in the use of hydrogen in the treatment of cancer. Although the field is still relatively new, molecular hydrogen appears to have promise as an adjuvant treatment for cancer patients, and may also have a role in prevention medicine for high risk populations.

\section{Conflict of Interests}

The authors declare that they have no conflicts of interest.

\section{Funding}

This study was supported by the Major State Basic Research Development Program (2015CB856302,
2015CB553602), the National Natural Science Foundation of China (31870848, 81741110, 81802787), and the Natural Science Foundation of Shaanxi (2018JZ3005).

\section{References}

1. Ohta S. Recent progress toward hydrogen medicine: Potential of molecular hydrogen for preventive and therapeutic applications. Curr Pharm Des 2011;17(22):2241-52.

2. Runtuwene J, Amitani H, Amitani M, Asakawa A, Cheng KC, Inui A. Hydrogen-water enhances 5-fluorouracil-induced inhibition of colon cancer. Peer J 2015;3:e859.

3. Dole M, Wilson FR, Fife WP. Hyperbaric hydrogen therapy: A possible treatment for cancer. Science 1975;190(4210):152-4.

4. Ohsawa I, Ishikawa M, Takahashi K, Watanabe M, Nishimaki K, Yamagata K, Katsura K, Katayama Y, Asoh S, Ohta S. Hydrogen acts as a therapeutic antioxidant by selectively reducing cytotoxic oxygen radicals. Nat Med 2007;13(6):688-94.

5. Ge L, Yang M, Yang NN, Yin XX, Song WG. Molecular hydrogen: a preventive and therapeutic medical gas for various diseases. Oncotarget 2017;8(60):102653-73.

6. Yang Q, Ji G, Pan R, Zhao Y, Yan P. Protective effect of hydrogen-rich water on liver function of colorectal cancer patients treated with mFOLFOX6 chemotherapy. Mol Clin Oncol 2017;7(5):891-6.

7. Nonaka Y, Iwagaki H, Kimura T, Fuchimoto S, Orita K. Effect of reactive oxygen intermediates on the in vitro invasive capacity of tumor cells and liver metastasis in mice. Int J Cancer 1993;54(6):983-6.

8. Nakashima-Kamimura N, Mori T, Ohsawa I, Asoh S, Ohta S. Molecular hydrogen alleviates nephrotoxicity induced by an anti-cancer drug cisplatin without compromising anti-tumor activity in mice. Cancer Chemother Pharmacol 2009;64(4):753-61.

9. Gao Y, Yang H, Fan Y, Li L, Fang J, Yang W. Corrigendum to "Hydrogen-rich saline attenuates cardiac and hepatic injury in doxorubicin rat model by inhibiting inflammation and apoptosis". Mediators Inflamm 2017;2017:3675910.

10. Terasaki Y, Suzuki T, Tonaki K, Terasaki M, Kuwahara N, Ohsiro J, Iketani M, Takahashi M, Hamanoue M, Kajimoto Y, Hattori S, Kawaguchi H, Shimizu A, Ohsawa I. Molecular hydrogen attenuates gefitinib-induced exacerbation of naphthalene-evoked acute lung injury through a reduction in oxidative stress and inflammation. Lab Invest 2019;99(6):793-806.

11. Mihailovic M, Milosevic V, Grigorov I, Poznanovic G, Ivanovic-Matic S, Grdovic N, Bogojevic D. The radioprotective effect of alpha2-macroglobulin: a morphological study of rat liver. Med Sci Monit 2009;15(7):BR188-93.

12. Chuai Y, Zhao L, Ni J, Sun D, Cui J, Li B, Qian L, Gao F, Cai J. A possible prevention strategy of radiation pneumonitis: combine radiotherapy with aerosol inhalation of hydrogen-rich solution. Med Sci Monit 2011;17(4):HY1-4.

13. Xiao HW, Li Y, Luo D, Dong JL, Zhou LX, Zhao SY, Zheng QS, Wang HC, Cui M, Fan SJ. Hydrogen-water ameliorates radiation-induced gastrointestinal toxicity via MyD88's effects on the gut microbiota. Exp Mol Med 2018;50:e433.

14. Kang KM, Kang YN, Choi IB, Gu Y, Kawamura T, Toyoda Y, Nakao A. Effects of drinking hydrogen-rich water on the quality of life 
of patients treated with radiotherapy for liver tumors. Med Gas Res 2011;1(1):11.

15. Mei K, Zhao S, Qian L, Li B, Ni J, Cai J. Hydrogen protects rats from dermatitis caused by local radiation. J Dermatolog Treat 2014;25(2):182-8

16. Zhang B, Zhao Z, Meng X, Chen H, Fu G, Xie K. Hydrogen ameliorates oxidative stress via PI3K-Akt signaling pathway in UVB-induced HaCaT cells. Int J Mol Med 2018;41(6):3653-61.

17. Zhao P, Jin Z, Chen Q, Yang T, Chen D, Meng J, Lu X, Gu Z, He Q. Local generation of hydrogen for enhanced photothermal therapy. Nat Commun 2018;9(1):4241.

18. Chen Y, Zong C, Guo Y, Tian L. Hydrogen-rich saline may be an effective and specific novel treatment for osteoradionecrosis of the jaw. Ther Clin Risk Manag 2015;11:1581-5.

19. Saitoh Y, Okayasu H, Xiao L, Harata Y, Miwa N. Neutral pH hydrogen-enriched electrolyzed water achieves tumor-preferential clonal growth inhibition over normal cells and tumor invasion inhibition concurrently with intracellular oxidant repression. Oncol Res 2008;17(6):247-55.

20. Kawai D, Takaki A, Nakatsuka A, Wada J, Tamaki N, Yasunaka T, Koike K, Tsuzaki R, Matsumoto K, Miyake Y, Shiraha H, Morita M, Makino H, Yamamoto K. Hydrogen-rich water prevents progression of nonalcoholic steatohepatitis and accompanying hepatocarcinogenesis in mice. Hepatology 2012;56(3):912-21.

21. Li Q, Tanaka Y, Miwa N. Influence of hydrogen-occluding-silica on migration and apoptosis in human esophageal cells in vitro. Med Gas Res 2017;7(2):76-85.

22. Akagi J, Baba H. Hydrogen gas restores exhausted $\mathrm{CD} 8^{+} \mathrm{T}$ cells in patients with advanced colorectal cancer to improve prognosis. Oncol
Rep 2018;41(1):301-11

23. Wang D, Wang L, Zhang Y, Zhao Y, Chen G. Hydrogen gas inhibits lung cancer progression through targeting SMC3. Biomed Pharmacother 2018;104:788-97.

24. Jiang Y, Liu G, Zhang L, Cheng S, Luo C, Liao Y, Guo S. Therapeutic efficacy of hydrogen-rich saline alone and in combination with PI3Kinhibitor in nonsmall cell lung cancer. Mol Med Rep 2018;18(2):2182-90

25. Wang P, Jia L, Chen B, Zhang L, Liu J, Long J, Li Y. Hydrogen inhalation is superior to mild hypothermia in improving cardiac function and neurological outcome in an asphyxial cardiac arrest model of rats. Shock 2016;46(3):312-8.

26. Hou C, Wang Y, Zhu E, Yan C, Zhao L, Wang X, Qiu Y, Shen H, Sun X, Feng Z, Liu J, Long J. Coral calcium hydride prevents hepatic steatosis in high fat diet-induced obese rats: A potent mitochondrial nutrient and phase II enzyme inducer. Biochem Pharmacol 2016;103:8597.

27. Hou C, Peng Y, Qin C, Fan F, Liu J, Long J. Hydrogen-rich water improves cognitive impairment gender-dependently in APP/PS1 mice without affecting A $\beta$ clearance. Free Radic Res 2018;52(11-12):131122.

28. Asada R, Kageyama K, Tanaka H, Matsui H, Kimura M, Saitoh Y, Miwa N. Antitumor effects of nano-bubble hydrogen-dissolved water are enhanced by coexistent platinum colloid and the combined hyperthermia with apoptosis-like cell death. Oncol Rep 2010;24(6):1463-70. 29. Yang Y, Zhu Y, Xi X. Anti-inflammatory and antitumor action of hydrogen via reactive oxygen species. Oncol Lett 2018;16(3):2771-6. 30. Ostojic SM. Molecular hydrogen: an inert gas turns clinically effective. Ann Med 2015;47(4):301-4. 